\title{
NEW BEREZIN SYMBOL INEQUALITIES FOR OPERATORS ON THE REPRODUCING KERNEL HILBERT SPACE
}

\author{
RAMIZ TAPDIGOGLU
}

Abstract. We use Kittaneh and Manasrah inequality and Kian's functional calculus method to prove some new inequalities for Berezin symbols and Berezin numbers of operators. In particular, we prove that

$$
\operatorname{ber}\left(f(A)^{2}\right) \leqslant \operatorname{ber}\left(\frac{f(A)^{p}}{p}+\frac{f(A)^{q}}{q}\right)
$$

for all self-adjoint operators $A$ on the reproducing kernel Hilbert space $\mathscr{H}(\Omega)$ with spectrum in $J \subset(-\infty,+\infty)$ and all continuous nonnegative functions $f$ defined on $J$. We also prove new upper and lower bounds for Berezin numbers of reproducing kernel Hilbert space operators. Among our results, we prove that if $A: \mathscr{H}(\Omega) \rightarrow \mathscr{H}(\Omega)$ is a bounded pseudo-hypornormal operator on the reproducing kernel Hilbert space $\mathscr{H}(\Omega)$, then for all non-negative non-decreasing pseudo-operator convex function $f$ on $[0, \infty)$, we have

$$
f(\operatorname{ber}(A)) \leqslant \frac{1}{2}\left\|f\left(\frac{|A|}{1+\frac{\xi_{|A|}^{2}}{8}}\right)+f\left(\frac{\left|A^{*}\right|}{1+\frac{\xi_{|A|}^{2}}{8}}\right)\right\|_{\mathrm{Ber}},
$$

where $\|\cdot\|_{\text {Ber }}$ denotes the Berezin norm of operator.

Mathematics subject classification (2020): 47B35.

Keywords and phrases: Reproducing kernel Hilbert space, Berezin symbol, Berezin number, Berezin norm, self-adjoint operator, functional calculus.

\section{REFERENCES}

[1] Y. Al-Manasrah And F. Kittaneh, A generalization of two refined Young inequalities, Posivity, 19 (2015), 757-768.

[2] N. AronZajn, Theory of reproducing kernels, Trans. Amer. Math. Soc., 68 (1950), 337-404.

[3] M. BAKHerad, R. LashKaripoor, M. Hajmdnamadi And U. YAMAnci, Complete refinements of the Berezin number inequalities, arXiv: 2003.098226v1[math.FA] 22 Mar 2020.

[4] M. BAKHERAD AND M. GARAYEV, Berezin number inequalities for operators, Concrete Operators, 6 (1) (2019), 33-43.

[5] H. BAŞARAN, M. GÜRdAl AND A. N. GÜNCAN, Some operator inequalities associated with Kantorovich and Hölder-McCarthy inequalities and their applications, Turkish J. Math., 43 (1) (2019), $523-532$.

[6] F. A. BEREZIN, Covariant and contravariant symbols for operators, Math. USSR-Izv., 6 (1972), $1117-1151$.

[7] M. EnGLIŚ, Compact Toeplitz operators via the Berezin transform on bounded symmetric domains, Integr. Equat. Oper. Theory, 33 (1999), 426-455.

[8] M. T. Garayev, The Berezin number, Norm of Hankel Operator and Related Topics, Operator Theory: Advances and Applications, 247 (2015), 87-100.

[9] M. T. GARAYEV, Berezin symbols, Hölder-McCarthy and Young inequalities and their applications, Proc. Inst. Math. Mech. Natl. Acad. Sci. Azerb., 43 (2) (2017), 287-295. 
[10] M. T. Garayev, H. Guediri And H. SAdRAOUI, Applications of reproducing kernels and Berezin symbols, New York J. Mathematics, 22 (2016), 583-604.

[11] M. T. GARAYEV, M. GÜRDAl AND A. OKUDAN, Hardy-Hilbert's inequality and power inequalities for Berezin numbers of operators, Math. Inequal. Appl., 19 (3) (2016), 883-891.

[12] M. T. GARAYEV, M. GÜRDAL AND S. SAlTAN, Hardy type inequality for reproducing kernel Hilbert space operators and related problems, Positivity, 21 (4) (2017), 1615-1623.

[13] M. T. Garayev, S. Saltan, F. Beuzaffour and B. Aktan, Some inequalities involving Berezin symbols of operator means and related questions, Rev. R. Acad. Cienc. Exactas Fís. Nat. Ser. A Math. RACSAM, 114 (85) (2020), 1-17.

[14] B. Gustafson And M. Putinar, Hyponormal Quantizastion of Planar Domains, Lecture Notes in Mathematics, 2199, doi:10.1007/978-3-319-65810-0, Springer International Publishing AG 2017.

[15] P. R. Halmos, A Hilbert space problem book, Series: Graduate Texts in Mathematics, vol. 19., New York, 1982.

[16] M. T. KARAEV, Berezin symbol and invertibility of operators on the functional Hilbert spaces, J. Funct. Anal., 238 (2006), 181-192.

[17] M. T. KARAEV, Reproducing kernels and Berezin symbols techniques in various questions of operator theory, Complex Anal. Oper. Theory, 7 (4) (2013), 983-1018.

[18] T. Kato, Notes on some inequalities for linear operators, Math. Ann., 125 (1952), 208-212.

[19] M. KiAn, Operator Jensen inequality for superquadratic functions, Linear Algebra Appl., 456 (2014), $82-87$.

[20] M. KIAN, Hardy-Hilbert type inequalities for Hilbert space operators, Ann. Funct. Anal., 3 (2012), $128-134$.

[21] F. KitTANEH, A numerical radius inequality and an estimate for the numerical radius of the Frobenius companion matrix, Studia Math., 158 (2003), 11-17.

[22] F. Kittaneh And Y. ManasRah, Improved Young and Heinz inequalities for matrices, J. Math. Anal. Appl., 361 (2010), 262-269.

[23] J. MiaO And D. Zheng, Compact operators on Bergman spaces, Integr. Equat. Oper. Theory, 48 (2004), 61-79.

[24] E. Nordgren And P. Rosenthal, Boundary values of Berezin symbols, Oper. Theory: Advances and Applications, 73 (1994), 362-368.

[25] M. E. OmidVAR, H. R. Moradi AND K. Shebrawi, Sharpening some classical numerical radius inequalities, Operators and Matrices, 12(2) (2018), 407-416.

[26] W. REID, Symmetrizable completely continuous linear transformations in Hilbert Space, Duke Math., 18 (1951), 41-56.

[27] U. YAMANCI AND M. GÜRDAL, On numerical radius and Berezin number inequalities for reproducing kernel Hilbert space, New York J. Mathematics, 23 (2017), 1531-1537.

[28] U. YAMANCI, M. GÜRDAL AND M. T. GARAYEV, Berezin number inequality for convex function in reproducing kernel Hilbert space, Filomat, 31 (18) (2017), 5711-5717.

[29] U. YAMANCI, M. T. GARAYEV AND C. ÇELIK, Hardy-Hilbert type inequality in reproducing kernel Hilbert space: its applications and related results, Linear Multilinear Algebra, 67 (4) (2019), 830-842.

[30] L. ZOU AND Y. JiAnG, Improved arithmetic-geometric mean inequality and its application, J. Math. Inequal., 9 (2015), 107-111. 